\title{
Facile Photoreduction of a Heme Peptide Encapsulated in Nanostructured Materials
}

\author{
B. Paul Zhang, ${ }^{\dagger}$ Michael T. Janicke, ${ }^{\dagger}$ William H. Woodruff, ${ }^{\dagger}$ and James A. Bailey \\ * \\ Bioscience Division (B-4, Mail Stop J586), ${ }^{\dagger}$ Chemistry Division (C-SIC, Mail Stop J514) \\ Los Alamos National Laboratory, Los Alamos, New Mexico 87545
}

\section{Synthesis of MCM-41}

$1.61 \mathrm{~g}$ of cetyltrimethylammonium bromide was dissolved in a polypropylene bottle by adding $9.99 \mathrm{~g}$ of $2 \mathrm{M} \mathrm{NaOH}$ solution and $78.46 \mathrm{~g}$ of deionized water and then gently heating the bottle at $40^{\circ} \mathrm{C}$ for 10 min. On dissolution of the surfactant, $7.70 \mathrm{~g}$ of Tetraethoxysilane (TEOS) was added to the solution dropwise. After stirring the mixture for $22 \mathrm{hrs}$ at room temperature, the precipitate was centrifuged and washed with deionized water 5 times. The solid was dried at $110^{\circ} \mathrm{C}$ overnight followed by heating it with $80 \mathrm{ml}$ of deionized water in a $100 \mathrm{ml}$ of Parr bomb at $110^{\circ} \mathrm{C}$ for 7 days. The solid was recovered by centrifuging. It was rinsed with deionized water 3 times. The solid was dried in an oven for a few hours before put into a furnace. The surfactant was removed by slowly heating the solid from room temperature to $520^{\circ} \mathrm{C}$ under dried air and the temperature was held there for 6hrs before the heating was stopped. $0.73 \mathrm{~g}$ of white solid was obtained.

\section{Synthesis of SBA-15}

$3.46 \mathrm{~g}$ of Pluronic123 (BASF, P123) was dissolved in $4.5 \mathrm{~g}$ of concentrated $\mathrm{HCl}$ and $21.6 \mathrm{~g}$ of water. Upon dissolution, sodium silicate solution (7.51 g containing $28.56 \% \mathrm{SiO}_{2}$, from the PQ Corporation, was diluted by $30.04 \mathrm{~g}$ of deionized water) was added. The solution was heated in a polypropylene bottle in a $35-^{\circ} \mathrm{C}$ water bath while gently shaking for $24 \mathrm{hrs}$. It was then transferred to a Parr bomb and heated at $100{ }^{\circ} \mathrm{C}$ for $24 \mathrm{hrs}$. The solid was separated by centrifugation and dried at $100{ }^{\circ} \mathrm{C}$ overnight without washing. It was then slurred with $120 \mathrm{ml}$ ethanol with a few drops of concentrated $\mathrm{HCl}$ for 30 min. After centrifuging and rinsing with ethanol, the solid was dried at $110^{\circ}$ for 2 hrs. The removal of surfactant was carried out by calcining the solid under dried air slowly to $550^{\circ} .2 .03 \mathrm{~g}$ of white solid was obtained.

in situ functionalization of SBA-15, 5\% functional group loading

$2 \mathrm{~g}$ of P123 was dissolved in $62.5 \mathrm{~g}$ of $2 \mathrm{M} \mathrm{HCl}$. After the surfactant was dissolved, $4.05 \mathrm{~g}$ (0.0194 mol) of TEOS was added. The solution was heated gently and shaken in a $40-{ }^{\circ} \mathrm{C}$ water bath for $1 \mathrm{hr}$ before $0.2315 \mathrm{~g}$ ( $0.00105 \mathrm{~mol}) 3$-aminopropyltriethoxysilane (APTES) was added. The mixture was stirred for 15 min at room temperature before the bottle containing the mixture was taken back to the water bath. The mixture was gently shaken and heated at $40{ }^{\circ} \mathrm{C}$ for $20 \mathrm{hrs}$. Afterwards the polypropylene bottle was transferred into a $100^{\circ} \mathrm{C}$ oven and held at this temperature for $20 \mathrm{hrs}$. The solid was separated by centrifuging and air dried overnight. The surfactant removal was done via solvent extraction by putting the solid in a thimble in a Soxhlet apparatus and refluxing with $220 \mathrm{ml}$ of ethanol for $24 \mathrm{hr} .1 .17 \mathrm{~g}$ of white solid was obtained after drying in air.

Sample Characterization

X-ray powder diffraction (XRD) data were acquired on a SCINTAG XDS2000 powder diffractometer using $\mathrm{Cu} \mathrm{K}_{\alpha}$ radiation. Figure S1 shows XRD of MCM-41 sample. It is a typical hexagonal pattern and 
can be indexed as shown in the Figure S1. The cell parameter calculated from the position of the (100) reflection is $5.0 \mathrm{~nm}$. Since the wall thickness for MCM-41 is commonly around 1 to $1.5 \mathrm{~nm}$, the pore size of MCM-41 is around $3.7 \mathrm{~nm}$. Figure S2 is the XRD for SBA-15 sample. The three well-resolved peaks can be indexed as (100), (110), and (200) associated with hexagonal symmetry. The first peak $\left(d_{001}\right)$ is $9.4 \mathrm{~nm}$ calculated based on Bragg's law. The cell parameter is $10.8 \mathrm{~nm}$. The wall thickness for SBA-15 sample under this reaction condition is around $4 \mathrm{~nm}$ and is substantially thicker than the one for MCM-41 sample. The pore size of this sample is estimated around $7 \mathrm{~nm}$. Figure S3 is the XRD for propyl-amine functionalized SBA-15 sample. Its pattern is similar to the parent non-functionalized SBA-15 sample but is not as crystalline as the latter one because of the phase disorder due to introduction of organic silica. Since the first peak for the functionalized sample is at the same position as the non-functionalized one, they should have same cell parameter but the pore size of the functionalized sample should be reduced accordingly due to the incorporation of functional groups.

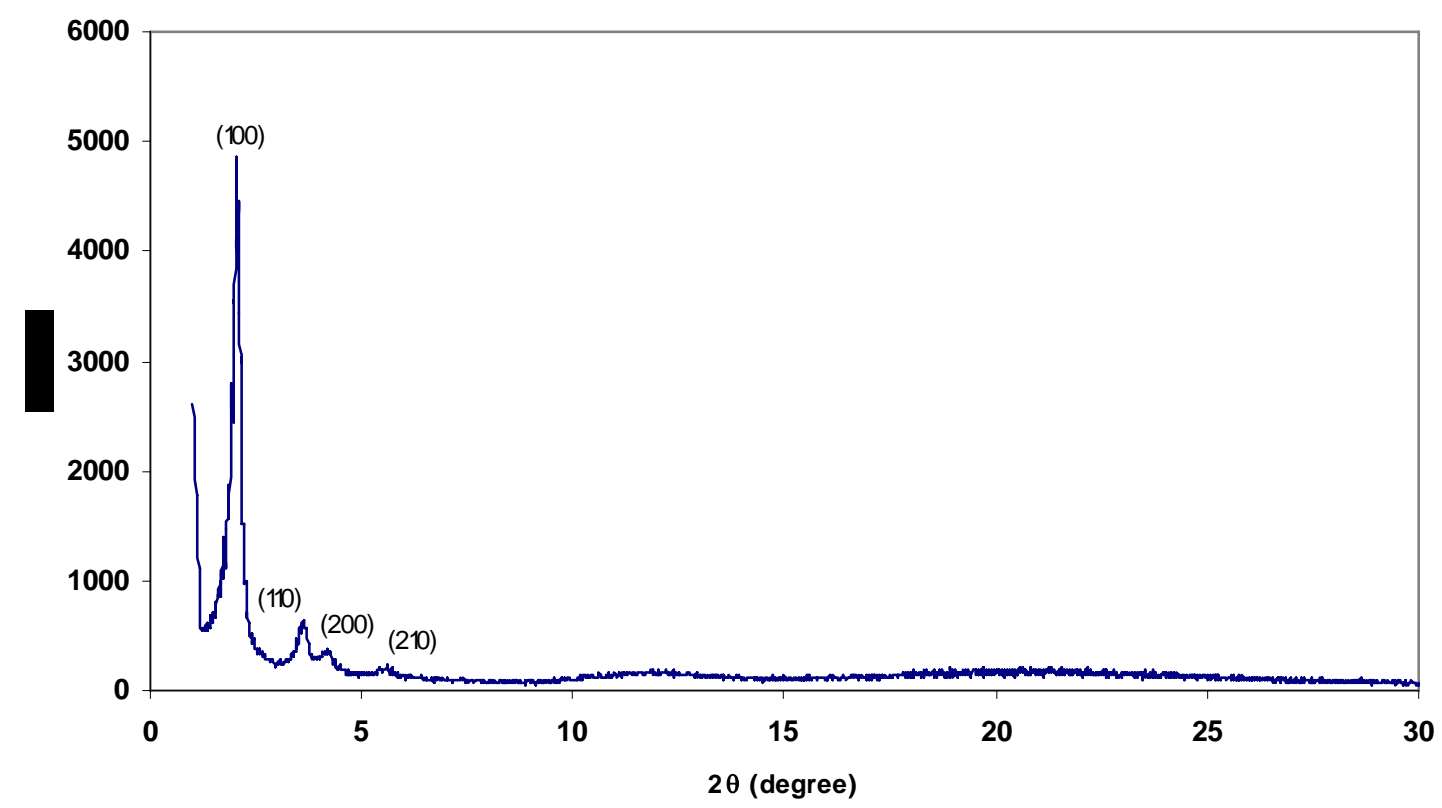

Figure S1. XRD pattern of MCM-41 


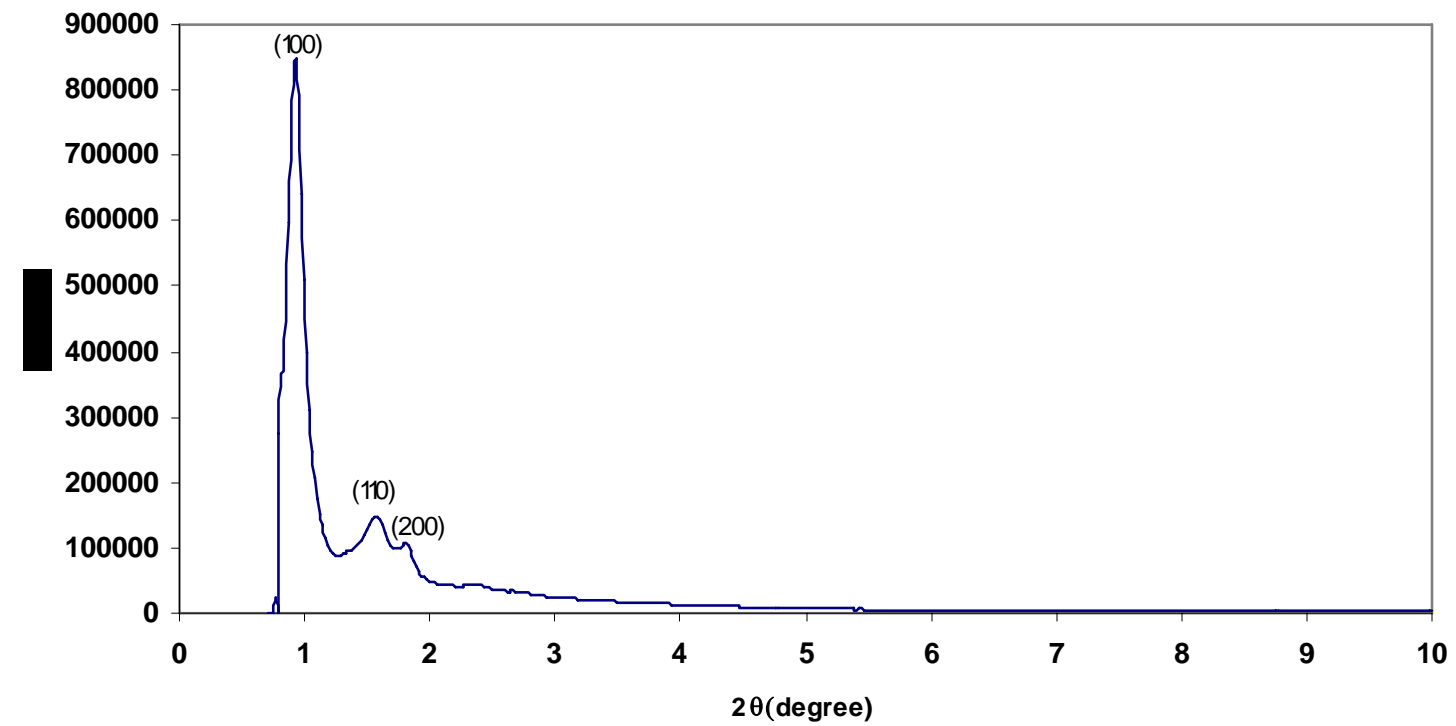

Figure S2. XRD pattern of SBA-15

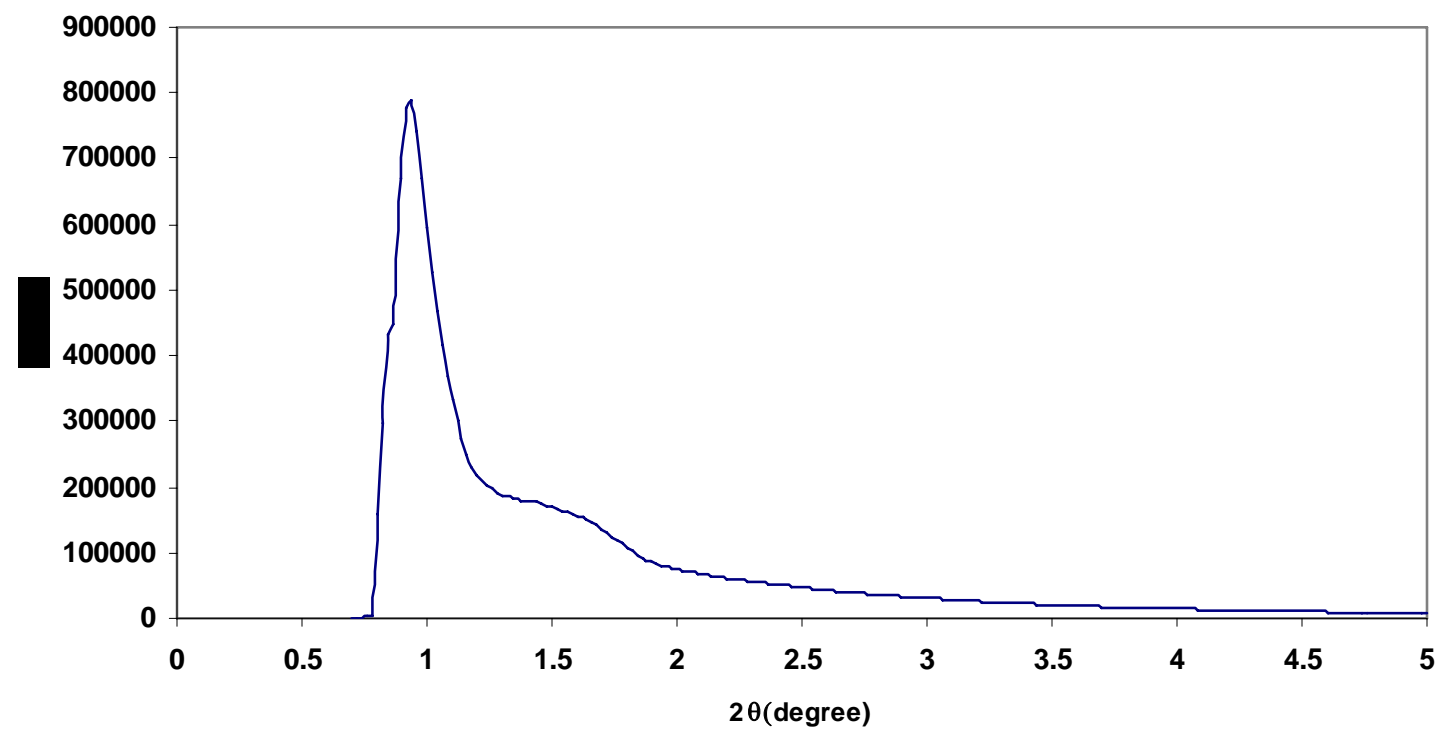

Figure S3. XRD Pattern of propyl-amine functionalized SBA-15(5\% loading) 


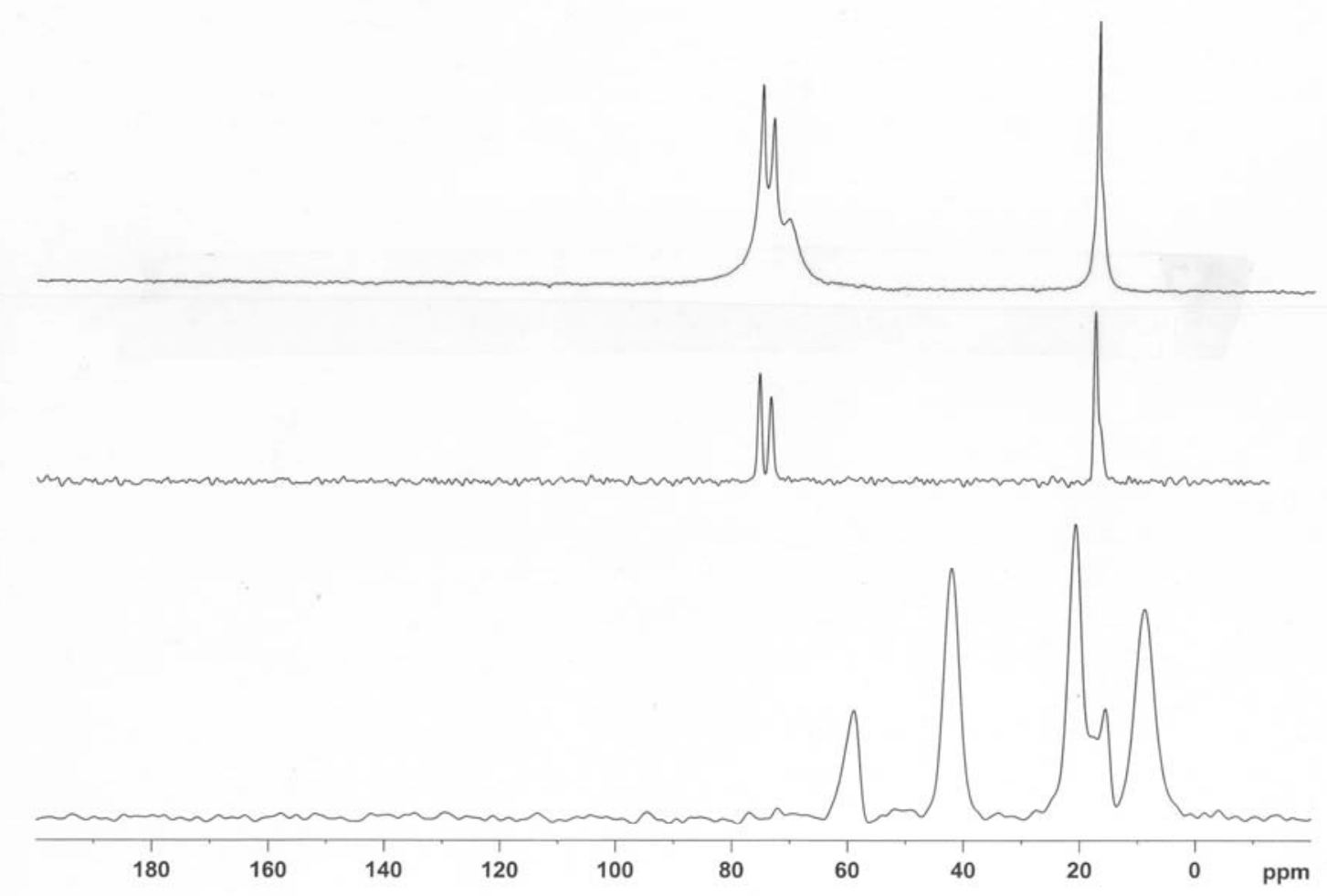

Figure S4. ${ }^{13} \mathrm{C}$ Solid-State Magic-angle spinning (MAS) NMR spectra for SBA-15 materials. (Top) SBA-15 material as-synthesized (before Soxhlet extraction of template) ${ }^{13} \mathrm{C}$ MAS spectrum with ${ }^{1} \mathrm{H}$ decoupling. (Middle) SBA-15 material as-synthesized ${ }^{13} \mathrm{C}$ cross-polarization MAS (CPMAS) spectrum. (Bottom) AFS material ${ }^{13} \mathrm{C}$ CPMAS spectrum after Soxhlet extraction. The resonances due to Pluronic 123 at about 17 ppm and between 70-75 ppm are readily apparent in the upper two traces, while the lower trace shows Soxhlet extraction is highly efficient at removing Pluronic 123 such that the 70-80 ppm resonances are below detection limits. The remaining resonances in the lower trace arise from the amino-propyl functionalization (9, 21, and $42 \mathrm{ppm}$ ), and ethanol used in the Soxhlet extraction (16 and $59 \mathrm{ppm}$ ).

The data was collected on a Bruker Avance $400 \mathrm{MHz}$ wide-bore system with a $4 \mathrm{~mm}$ rotor and the samples were spun at $10,000 \mathrm{~Hz}$. Typically $4.25 \mu \mathrm{m} 90^{\circ}$ pulses for the ${ }^{13} \mathrm{C}$ were used with a $3.5 \mathrm{~ms}$ contact time for cross-polarization. For the AFS material, a 3 s relaxation delay was used between acquisitions and a total of 16,800 scans were recorded. 